\title{
Structural, Dynamical, and Energetical Consequences of Rett Syndrome Mutation R133C in MeCP2
}

\author{
Tugba G. Kucukkal and Emil Alexov \\ Computational Biophysics and Bioinformatics, Department of Physics, Clemson University, Clemson, SC 29634, USA \\ Correspondence should be addressed to Tugba G. Kucukkal; tugbak@g.clemson.edu
}

Received 15 December 2014; Accepted 11 March 2015

Academic Editor: Volkhard Helms

Copyright (C) 2015 T. G. Kucukkal and E. Alexov. This is an open access article distributed under the Creative Commons Attribution License, which permits unrestricted use, distribution, and reproduction in any medium, provided the original work is properly cited.

\begin{abstract}
Rett Syndrome (RTT) is a progressive neurodevelopmental disease affecting females. RTT is caused by mutations in the MECP2 gene and various amino acid substitutions have been identified clinically in different domains of the multifunctional MeCP2 protein encoded by this gene. The R133C variant in the methylated-CpG-binding domain (MBD) of MeCP2 is the second most common disease-causing mutation in the MBD. Comparative molecular dynamics simulations of R133C mutant and wild-type MBD have been performed to understand the impact of the mutation on structure, dynamics, and interactions of the protein and subsequently understand the disease mechanism. Two salt bridges within the protein and two critical hydrogen bonds between the protein and DNA are lost upon the R133C mutation. The mutation was found to weaken the interaction with DNA and also cause loss of helicity within the 141-144 region. The structural, dynamical, and energetical consequences of R133C mutation were investigated in detail at the atomic resolution. Several important implications of this have been shown regarding protein stability and hydration dynamics as well as its interaction with DNA. The results are in agreement with previous experimental studies and further provide atomic level understanding of the molecular origin of RTT associated with R133C variant.
\end{abstract}

\section{Introduction}

Rett Syndrome (RTT) is an X-linked severe neurodevelopmental disorder [1-5]. It is a progressive disease after onset and especially affects the expressive language and hand use [6-10]. RTT affects 1 in 10,000 females with 20,000 RTT patients in the US and 50,000 worldwide [7]. The mutations in $\mathrm{MeCP} 2$ are the major cause of RTT as they have been detected in more than $90 \%$ of classical RTT patients $[8,11]$. In addition, the $\mathrm{MeCP} 2$ mutations are associated with $\mathrm{X}$-linked mental retardation and other neurological disorders $[8,12]$. $\mathrm{MeCP} 2$ is a member of the methyl-CpG-binding domain (MBD) family of proteins and has three major domains: the abovementioned $\mathrm{MBD}$, the transcriptional repression domain (TRD), and the C-terminal domain (CTD). MeCP2 binds to symmetrical methylated $5^{\prime} \mathrm{CpG}$ pairs through its MBD with a preference for A/T-rich motifs [13]. It also belongs to intrinsically disordered family of proteins [14] and therefore binds to a number of other partners through its disordered regions, which span about $65 \%$ of the protein [15]. In general, MeCP2 serves diverse functions in gene regulation and chromatin organization and particularly it is a transcriptional repressor that mediates gene silencing through binding to methylated DNA [11]. However, recent studies indicate that it also can act as an activator [11]. In the body, it is distributed to all tissues but particularly abundant in brain [11].

There have been a number of RTT-causing mutations identified at different regions of the 486-residue MeCP2 protein. Considering the mutations with a frequency of more than $0.05 \%$, the $20 \%$ of the RTT cases are caused by mutations in the MBD domain of MeCP2. Also, the deleterious mutations are responsible for $27 \%$ of the cases and the missense mutations in parts of $\mathrm{MeCP} 2$ other than the MBD domain are responsible for $14 \%$ of RTT cases [16].

Here we focus on the mutations occurring in MBD and particularly studied here is the R133C mutation [17, 18]. The Arg 133 is one of the two residues that make direct contacts 


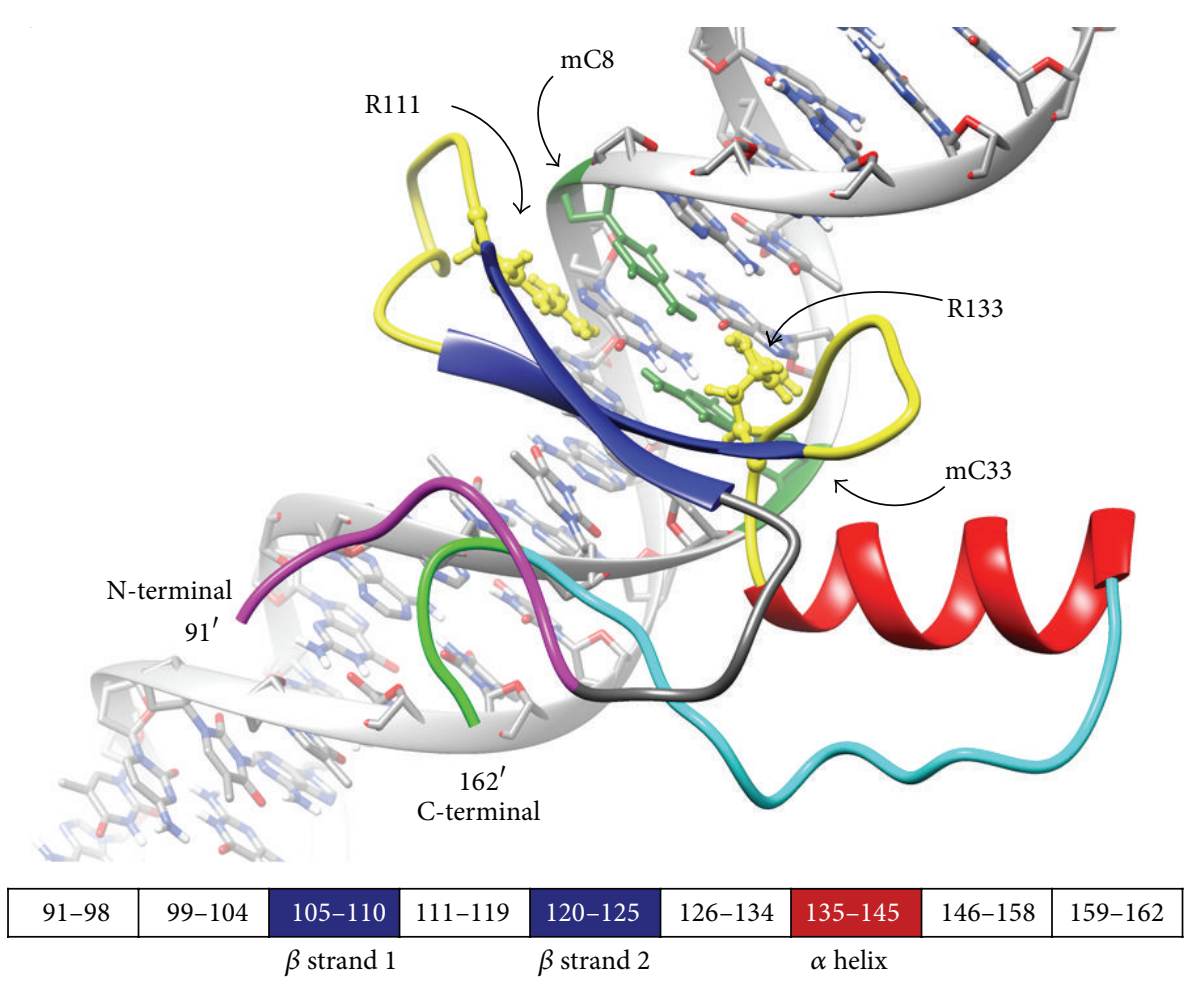

(a)

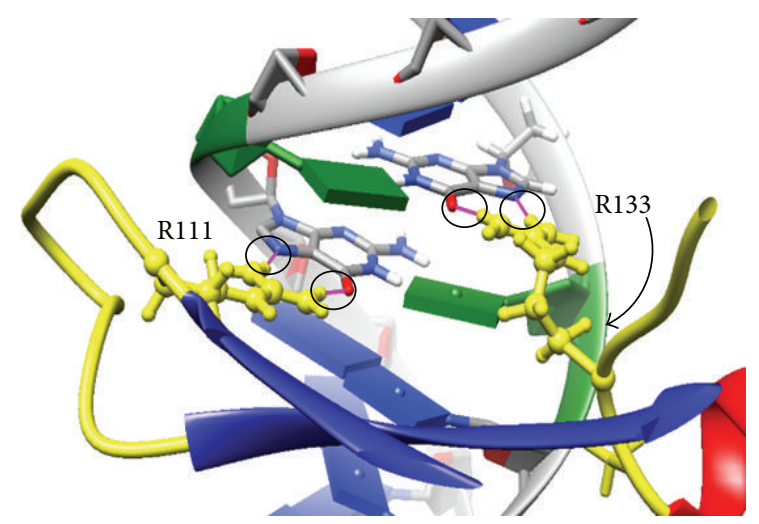

(b)

FIGURE 1: Initial structure of WT protein with DNA (PDB ID: 3C2I). The two Arg residues (R133 and R111) are shown in yellow. The two methylated Cyt residues of DNA are shown in green. The 9 regions of protein are colored differently and sequence breakdown with structured regions is shown at the middle panel. The hydrogen bonding between R133 and R111 and DNA is shown (magenta colored lines in circles) at the bottom image.

with DNA and R133C mutation is the second most common mutation in MBD affecting more than $4 \%$ of all RTT cases [16]. Extensive molecular dynamics (MD) studies have been performed on both wild-type (WT) and R133C mutant to understand the effects of mutation on protein structural, dynamical, and energetical properties at the molecular level. The comparative MD studies reveal important details on how $\mathrm{R} 133 \mathrm{C}$ impacts MBD and MBD-DNA recognition. The calculated effects are consistent with previously published experimental data while providing further atomic level details of the molecular origin of disease associated with the R133C variant.

\section{Methods}

2.1. Structure Preparation. The X-ray structure of MBD of MeCP2 bound to DNA, PDB ID 3C2I [19], was used as the initial structure. This structure has the mutation A140M; therefore, first the Met at position 140 was mutated back to Ala ensuring the canonical sequence for the MBD. Then, the R133C mutation was introduced on this structure. The WT and $\mathrm{R} 133 \mathrm{C}$ structures were then independently subjected to further preparation. First, the missing hydrogen atoms were added and then the structures were solvated with TIP3 water 
molecules [20] together with sodium and chloride ions to ensure $0.15 \mathrm{M}$ concentration and neutral systems. The ions were placed using the Autoionize Plugin in the VMD software [21]. A periodic box with edges extending at least $10 \AA$ from solute atoms was used, thus ensuring 3-4 hydration layers in each direction. The initial periodic cell size was about $80 \times$ $53 \times 78 \AA^{3}$ with $\sim 31,000$ atoms in each system. All mutations and other structural preparations were performed utilizing the VMD program [21].

2.2. Molecular Dynamics. Langevin dynamics with periodic boundary conditions was performed in the NPT (constant pressure, constant temperature) ensemble using the CHARMM22 force field for proteins [22] with CMAP corrections $[23,24]$ and the CHARMM27 force field $[25,26]$ for the DNA. vdW interactions were truncated with a switching function in $10 \AA$ distance with 8 to $10 \AA$ cutoff. Electrostatic interactions were truncated with particle mesh Ewald (PME) [27] and $1 \AA$ grid spacing was used. A temperature of $298 \mathrm{~K}$ was maintained using Langevin dynamics, with a damping constant of $1 \mathrm{ps}^{-1} .1 \mathrm{~atm}$ pressure was maintained using the Nosé-Andersen Langevin piston [28, 29]. First, the systems were relaxed for 4000 steps and then MD simulations were run with a time step of $1 \mathrm{fs}$ for $\sim 5 \mathrm{~ns}$. Then, a time step of $1.5 \mathrm{fs}$ was used and the simulations were run for $220 \mathrm{~ns}$ in total. The lengths of all bonds involving hydrogens were constrained with the RATTLE algorithm [30] as implemented in NAMD [31]. All simulations were performed using NAMD.

2.3. Analysis of MD Energies and Trajectories. All the analyses were performed utilizing VMD unless otherwise stated. Root mean square fluctuations (RMSF) of $C_{\alpha}$ atoms were calculated using the trajectory after the 30th ns (last $190 \mathrm{ns)}$ ensuring that the systems were equilibrated. The solvent accessible surface area (SASA) was calculated with a $1.4 \AA$ probe radius. Contact area was calculated as

$$
\text { Contact area }=\frac{\mathrm{SASA}_{\text {protein }}+\mathrm{SASA}_{\mathrm{DNA}}-\mathrm{SASA}_{\text {complex }}}{2} .
$$

Hydrogen bonding criteria used in analyses are the donoracceptor distance of $3 \AA$ or less and the donor-hydrogenacceptor angle of 20 degrees or less. Also, a $3.2 \AA$ cutoff of oxygen-nitrogen is used for salt bridges. The time-dependent distance profile of residue 133 and $\mathrm{mC} 33$ was calculated by taking the distance between center of mass of residue 133 of the protein and the center of mass mC33 of the DNA. All the analyses described so far have been performed using the structures saved every $3 \mathrm{ps}$.

Interaction energy (van der Waals and electrostatic) was calculated for structures saved every 200 ps with the same cutoff as the MD simulations. Cluster analysis was performed with a $2 \AA$ cutoff of RMSD for the residues 99 to 158. Secondary structure analysis was based on the DSSP algorithm [32] as implemented in the WHATIF program [33].

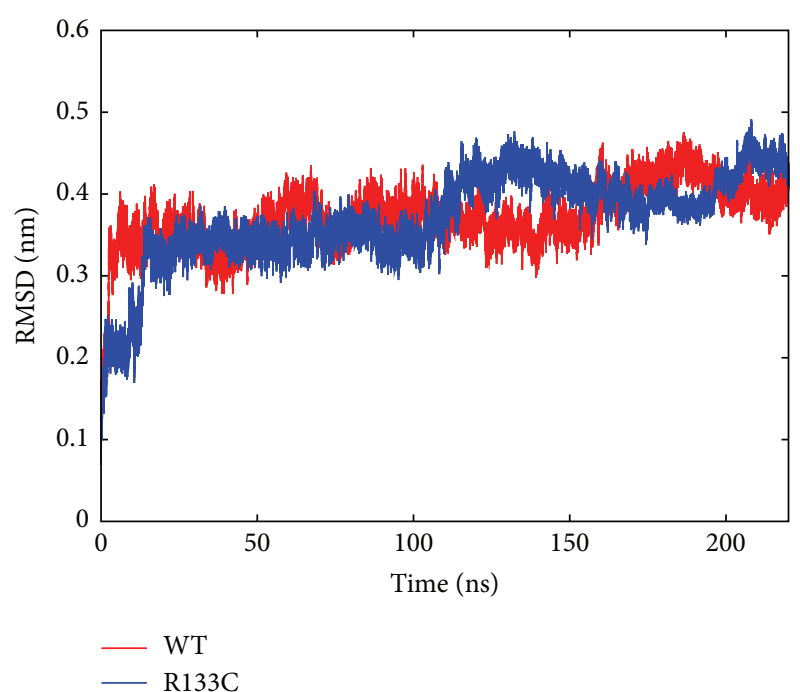

FIGURE 2: Time-dependent RMSD profile of residues 99-158 for WT (red) and R133C (blue).

\section{Results and Discussion}

The crystal structure of MBD of MeCP2 bound to DNA revealed important structural information regarding its binding to DNA as well as its fold. The protein has two major $\beta$ strands and an $\alpha$ helix forming a wedge-like shape with two major coil (turn) regions that make direct contacts with the two symmetrical methylated cytosine $(\mathrm{mC})$ of methylatedCpG DNA. As shown in Figure 1(a), the crystal structure indicates that the two Arg residues, Arg133 and Arg111, form direct hydrogen bonds with DNA. The frequent RTT mutation R133C has immediate structural consequences such as the loss of the direct hydrogen bond with DNA as shown in Figure 1(b). $220 \mathrm{~ns}$ long MD simulations of WT and R133C systems reveal important consequences of the mutation in structure, dynamics, and interactions of $\mathrm{MBD}$ of $\mathrm{MeCP} 2$.

The rest of the section is organized into three major components: (a) structural and dynamical analysis of the systems, (b) solvent accessible surface area (SASA) and radius of gyration $\left(R_{\text {gyr }}\right)$ analysis, and (c) analysis of the specific interactions of protein, DNA, and water through hydrogen bonding and energetics calculations.

(a) Impact of R133C Mutation on Structure and Conformational Dynamics. First, time-dependent root mean square deviations (RMSDs) of the MBD of MeCP2 (WT and R133C) from their initial structures were computed. Monitoring the RMSD evolution of the protein can give insights into its structural conformation and also the structural integrity of biomolecules in the simulation system. Based on the change in RMSD, the protein (MBD of MeCP2) sequence was divided into 9 regions. Not surprisingly, the coiled regions at the beginning and end of the sequence (91-98 and 159-162) were found to be excessively floppy; therefore the RMSD of the whole protein, Figure 2, was obtained by leaving these regions out. The RMSD change flattens after about $20 \mathrm{~ns}$ and the 

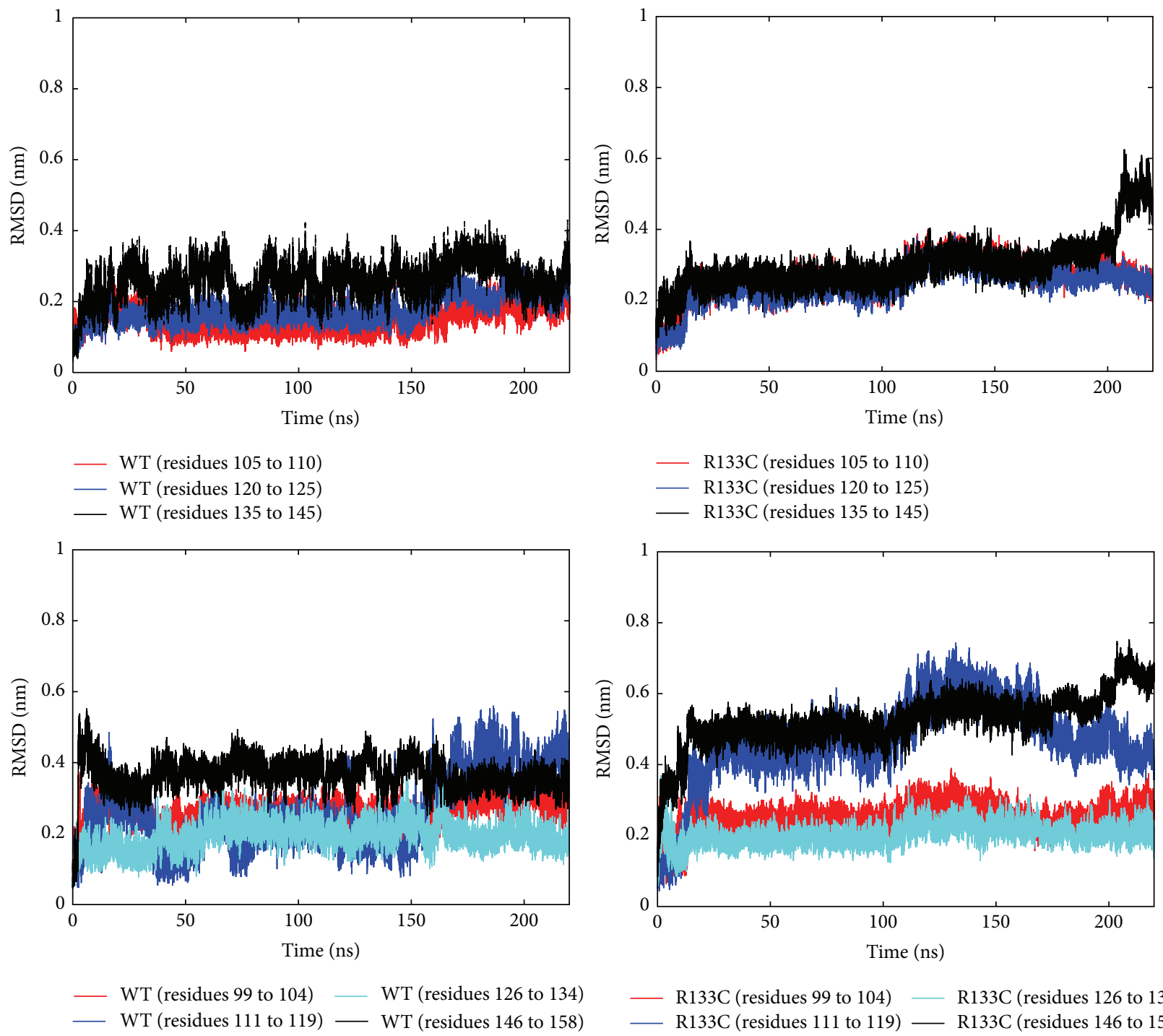

$\begin{array}{ll}\text { R133C (residues } 99 \text { to } 104) & \text { R133C (residues } 126 \text { to } 134) \\ -\mathrm{R} 133 \mathrm{C} \text { (residues } 111 \text { to } 119) & -\mathrm{R} 133 \mathrm{C} \text { (residues } 146 \text { to 158) }\end{array}$

FIgURE 3: Time-dependent RMSD changes of loop regions (bottom panels) and structured regions (top panels) for WT (left panels) and R133C (right panels).

variance stays under $0.1 \mathrm{~nm}$ until around 110 and $160 \mathrm{~ns}$ for WT and R133C, after which larger structural changes seem to take place. To pinpoint the source of these changes, the RMSD change over time for structured and coiled regions as shown in Figure 3 was examined. For the WT, the source of the changes was found to take place mostly in the coiled regions and visual inspection of MD trajectories reveals flipping of coiled regions. Although the R133C structure shows the same trend in general, it also has a drastic RMSD change in the helical region after $\sim 200 \mathrm{~ns}$.

The structures were examined more closely by performing cluster analysis (based on RMSD) for the WT and R133C MD trajectories for the sequence regions between 99 and 158. In each case, the structures were grouped in five (colored as yellow, purple, orange, green, and turquoise as shown in the first column of Figure 8) and representative structures were taken from each group. As the RMSD suggests in both cases, the structure remains the same for a long period of time ( 20-100 ns); therefore one of the groups in the cluster has a large number of structures (green), for which 4 representative structures were considered. Then, the representative structures were subjected to a secondary structure analysis based on the DSSP algorithm. These secondary structure analysis results are shown in Figure 8. Taken together with the time-dependent RMSD profiles, the origin of the possible structural change for the WT is the loss of a very short strand region (residues 131 and 132). However, the abrupt change in the RMSD profile of the helical region of R133C as shown in Figure 3 top right panel originates from a significant loss of helicity in the 142-144 region (Figure 8). Based on this analysis, unlike the WT, the mutant was found to have preserved the short strand region (residues 131 and 132). Having discussed the structural analysis, we note that the results are being reported from a single trajectory. 
The circular dichroism (CD) data indicated previously that the methylated DNA-induced stabilization for MBD of $\mathrm{MeCP} 2$ was less in the R133C mutant compared to WT [15]. In other words, the percent helicity was shown to increase $\sim 5 \%$ in WT upon binding to methylated DNA (mDNA); however, the R133C mutant MBD did not show this structural enhancement upon binding to mDNA. Also, experiments suggested that the R133C mutation caused the loss of specificity for mDNA but did not abolish binding altogether [15, 34] as seen in the current MD results. Therefore, our results suggesting loss of helicity in the presence of methylated DNA and maintenance of binding upon mutation are consistent with these previous findings about the secondary structure of MBD upon mutation.

It is quite possible that the loss of helicity or reduced mDNA-induced stabilization may originate from altered DNA-MBD interactions particularly at the mutation site 133. To verify this, the DNA-protein interactions were analyzed in detail in the last section.

Before moving on to altered interactions and SASA changes, another structural property of the protein, the salt bridges were monitored particularly in the vicinity within $6 \AA$ of R133 and within $6 \AA$ of R111. One salt bridge in each region was lost upon R133C mutation as seen in Table 1. We speculate that the loss of the salt bridge between E137 and R133 might contribute to the loss of helicity in 141-144 region. It is puzzling why the loss of helicity did not start at the beginning of the helical region particularly taking the loss of this salt bridge into consideration. We speculate that the significant change in the interactions of the 133 position might have been translated into the 141-144 region through an allosteric path. In addition, since $\mathrm{MeCP} 2$ is an intrinsically disordered protein, it is reasonable to expect the DNA to induce different structural features for the WT and mutant MBD based on the sequence difference. Therefore, this reduced helical content might also be reflecting a different binding mode of the mutant to mDNA.

Besides these structural changes, the dynamics of WT and $\mathrm{R} 133 \mathrm{C}$ proteins were assessed through root mean square fluctuations (RMSF) of $C_{\alpha}$ atoms of each residue (Figure 4). The RMSF profiles can be considered as a measure of the average atomic mobility and on this plot, the peaks indicate areas of protein that fluctuate the most during the simulation. The protein was found to be rigidified upon the R133C mutation in all regions except the helix region. The most pronounced reduction in flexibility was seen in the two coil regions between the strands and the helix. Interestingly, the last two residues of the helical region and the next two residues showed a significant increase in flexibility. This is consistent with the structural changes in that region as shown in an RMSD plot in Figure 3 (top right panel) and also the secondary structure analysis in Figure 8. Therefore, the R133C mutation was found to have a profound effect on the structure and dynamics of the helical part of the protein at long time scales.

(b) Impact of R133C Mutation on SASA and $R_{\text {gyr }}$. After examining the structural and dynamical properties of WT and $\mathrm{R} 133 \mathrm{C}$ proteins, the possible consequences of the $\mathrm{R} 133 \mathrm{C}$
TABLE 1: Salt bridges formed in the vicinity of R111 and R133 as monitored over the time-course of the simulations.

\begin{tabular}{lcc}
\hline & $\begin{array}{c}\text { Salt bridges near R133 and R111 } \\
\text { WT }\end{array}$ & R133C \\
\hline E137-R133 & $\checkmark$ & X \\
E137-K130 & $\checkmark$ & $\checkmark$ \\
D121-R111 & $\checkmark$ & $\checkmark$ \\
D121-K119 & $\checkmark$ & X \\
\hline
\end{tabular}

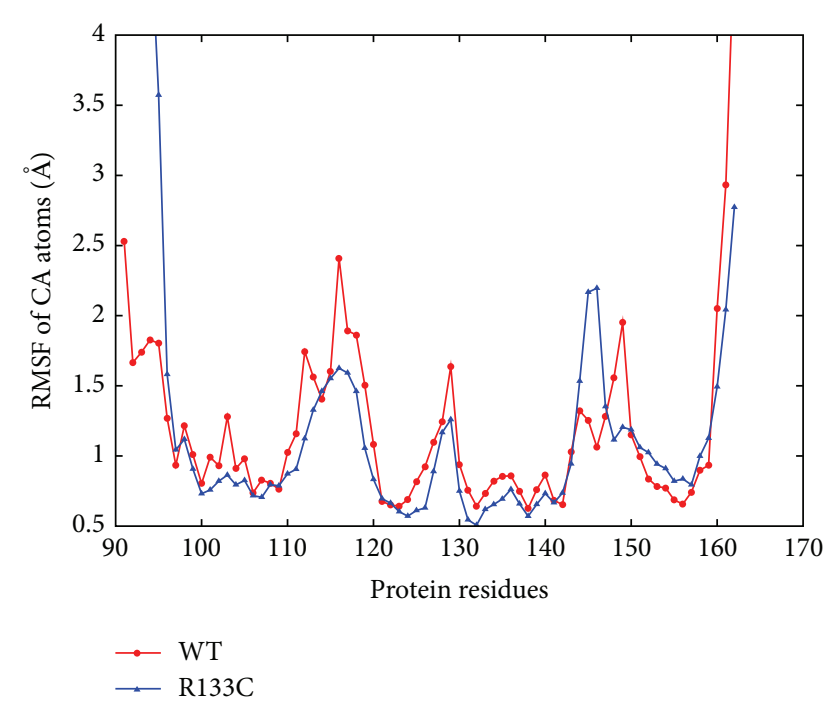

FIGURE 4: RMSF of CA atoms of all residues (91 to 162). Standard errors are omitted since they were negligibly small (on the order of $\left.10^{-3}\right)$.

mutation on hydration dynamics were monitored through solvent accessible surface area (SASA) profiles. Not surprisingly, the overall SASA profile for the proteins and the DNA have not been affected significantly by one residue change as shown in Figure 5 (top panels). However, the mutation caused a strong change in SASA of both the mutation position (133) and the methylated cytosine (mC33) of DNA Figure 5 (bottom panels). The visual inspection of the MD trajectories revealed that the coil region adapted a more buried conformation upon mutation. Interestingly, the R111 also became more buried as its SASA decreased by $23 \%$ upon mutation.

Following this, the compactness of the proteins was examined through computing their radius of gyration. Not surprisingly, the radius of gyration of the WT and R133C proteins did not show a significant change. Then, the two coil/turn regions in between strands and the helix were examined closely in regard to the radius of gyration profile. The 111-119 region did not show a significant change; however, as shown in Figure 5, the 126-134 region, in which the mutation took place, indicated an approximately $7 \%$ reduction in radius of gyration suggesting a more compact conformation for this coil region. This is consistent with the significant reduction of SASA in residue 133 upon mutation. 


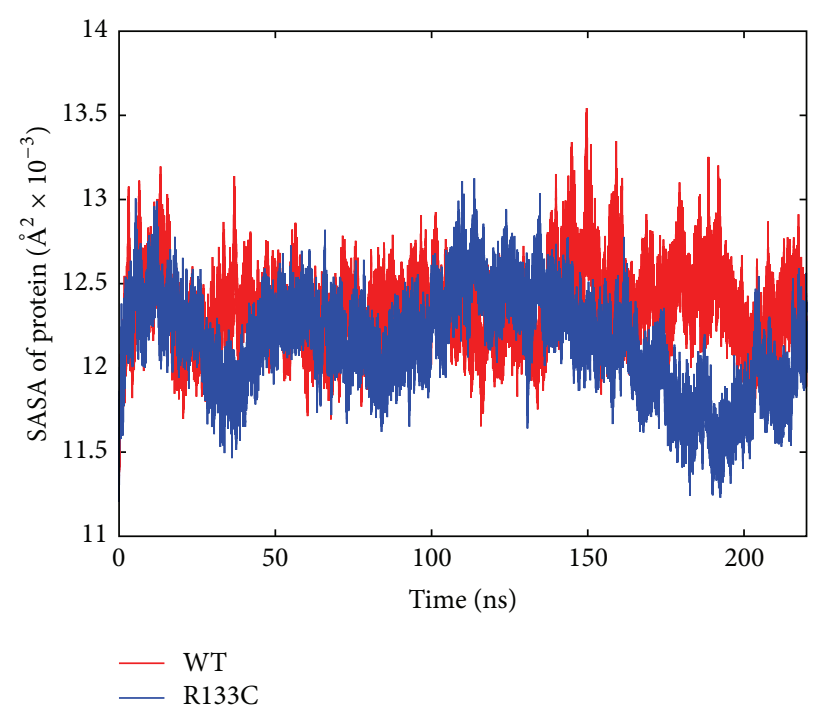

(a)

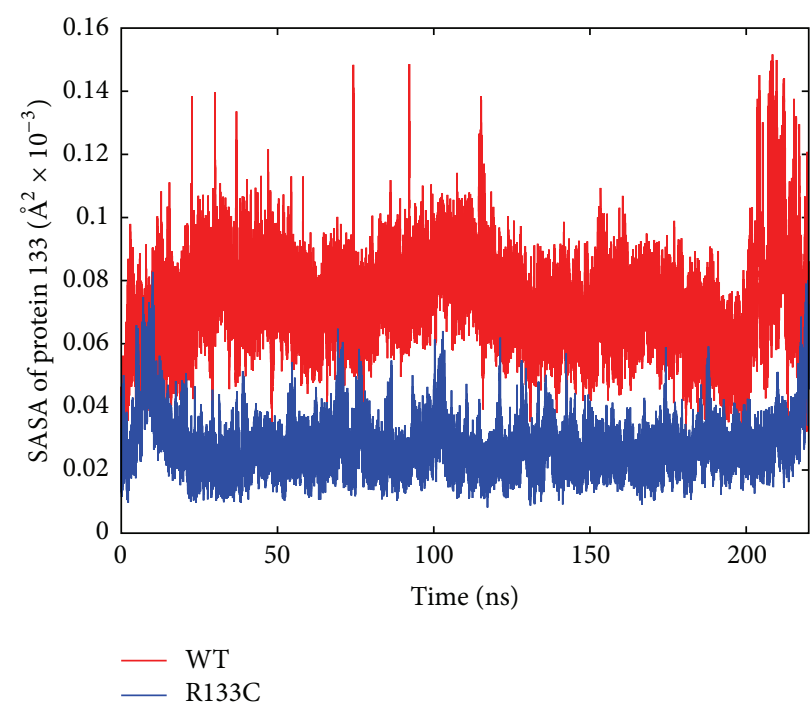

(c)

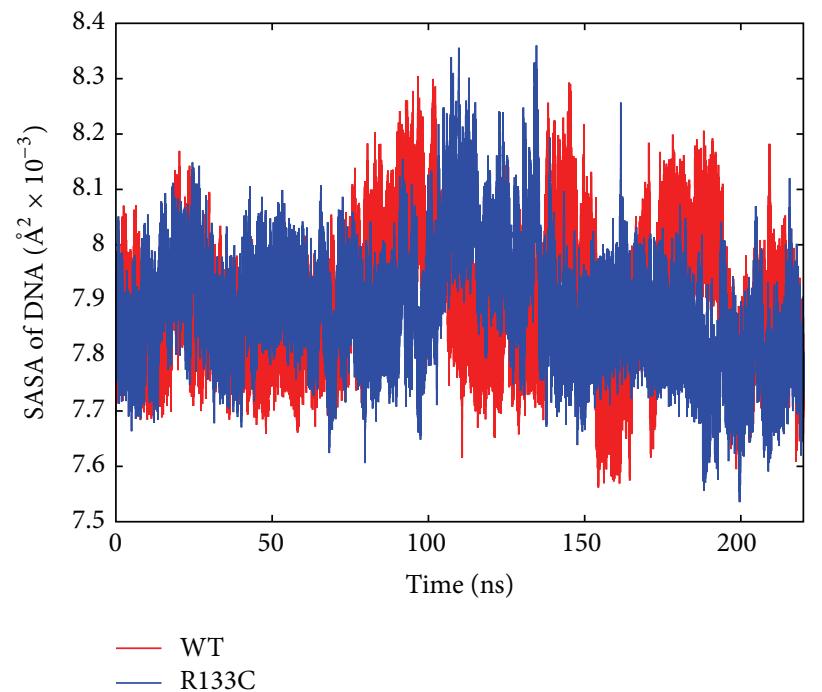

(b)

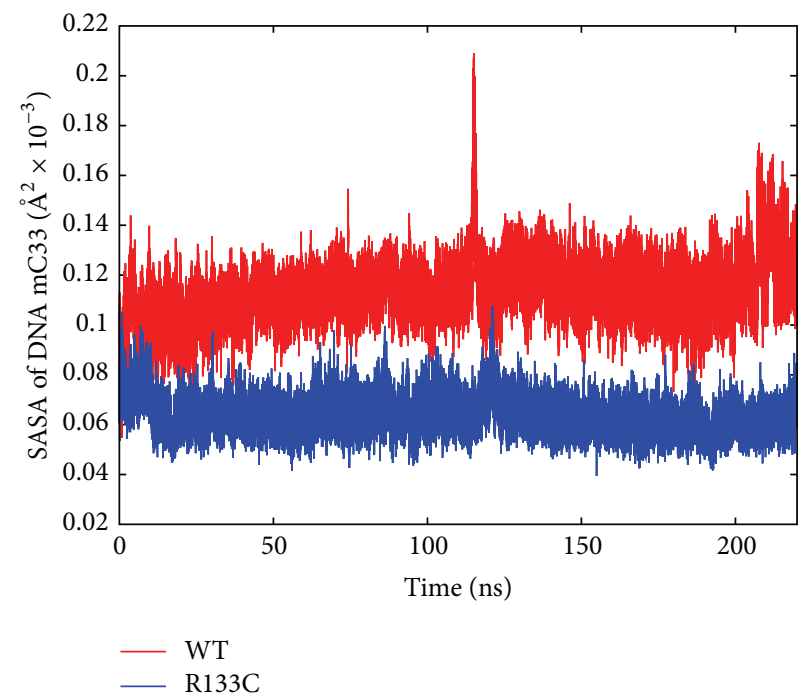

(d)

FIGURE 5: Time-dependent SASA profile of the full proteins (a) and residue 133 (c) and full DNA (b) and mC33 (d). WT is shown in red and $\mathrm{R} 133 \mathrm{C}$ is shown in blue.

(c) Impact of R133C Mutation on Interactions of MBD of $M e C P 2, D N A$, and Water. To quantify the significant SASA change of residue 133 further, the average number of hydrogen bonds that the proteins (WT and R133C) make with water was calculated. As shown in Table 2 (bottom table), the average number of hydrogen bonds in total decreased by $7 \%$ upon mutation. This overall decrease arose not only from the dramatic decrease (47\%) of hydrogen bonds that the residue 133 makes with water, but also from other residues. For example, another key Arg at position 111 makes 17\% less hydrogen bonds with water upon mutation of position 133 . Also consistent with this is the $23 \%$ reduction in SASA of residue 111 as previously mentioned.

Turning now to protein-DNA interactions and how they are affected by $\mathrm{R} 133 \mathrm{C}$ mutation, first, the hydrogen bonding network between the proteins and DNA was analyzed. The most obvious effect is that the direct hydrogen bond that Arg133 makes with the DNA is lost upon its mutation to Cys. Interestingly, the hydrogen bonding between R111 and DNA was also affected upon mutation indicating an $11 \%$ decrease. The degree of reduction in hydrogen bonding between the protein and DNA was found to be $21 \%$. Also, the timedependent contact area between the proteins (WT and R133C MBD) and DNA was monitored and an average overall decrease of $8 \%$ was observed in the R133C mutant system.

In addition, the time-dependent distance profile between residue 133 and $\mathrm{mC} 33$ of DNA indicated that the distance between the center of mass of residue 133 of protein and mC33 of DNA was lowered by about $11 \%(\sim 1 \AA)$. Therefore, geometrically, the Cys side chain is not sufficiently long to 
TABLE 2: Average number of hydrogen bonds formed with DNA (top) and water (bottom) computed over the course of the trajectories. Standard errors are omitted since they were negligibly small.

\begin{tabular}{lcc}
\hline & WT & R133C \\
\hline \multicolumn{3}{c}{ Average number of hydrogen bonds with DNA } \\
Protein & 7.21 & 5.70 \\
Residue 133 & 0.63 & 0.0 \\
Residue 111 & 0.74 & 0.66 \\
\hline \multicolumn{3}{c}{ Average number of hydrogen bonds with water } \\
Protein & 81.8 & 76.2 \\
Residue 133 & 1.68 & 0.79 \\
Residue 111 & 0.47 & 0.39 \\
DNA & 150 & 156 \\
mC33 & 2.70 & 2.73 \\
mC8 & 2.69 & 2.59 \\
\hline
\end{tabular}

get as close to DNA compared to Arg and also considering the more buried and compact conformation that the 126-134 loop region adapts, a significant decrease in the interaction of protein and the DNA is expected upon R133C mutation.

Finally, the interaction between the proteins and DNA, split into van der Waals and electrostatic interaction energy between DNA and proteins were calculated, as shown in Figure 7, to understand the manifestation of the loss of hydrogen bonding between the protein and DNA upon mutation. It should be clarified that the interaction energy is only a component of the total binding free energy and does not include desolvation penalty, for example. Consistent with the previous analyses, the R133C mutation was found to lower the interaction energy quite significantly. This is also consistent with previous EMSA (electrophoretic mobility shift assay) data suggesting a reduced binding of R133C to mDNA compared to WT [15]. The reduced interaction was found to be electrostatically driven as shown in Figure 6 and Table 3. The reduced interaction energy taken together with reduced hydrogen bonding between MBD and DNA might cause the reduced helicity in the R133C mutant. In other words, reduced interaction with the mDNA might cause reduced mDNA-induced stabilization and this may be manifested as the loss of helicity. Therefore, the R133C mutation was found to still maintain binding to DNA; however, its interaction is significantly reduced.

\section{Conclusion}

Comparative MD simulations of WT and mutant (R133C) $\mathrm{MBD}$ domain of MeCP2 were performed. The RTT mutation, $\mathrm{R} 133 \mathrm{C}$, was found to have pronounced effects on the structure and dynamics of $\mathrm{MBD}$ of the human $\mathrm{MeCP} 2$ protein and its interaction with DNA. The mutant was found to be stable and maintained binding to the methylated DNA, which is in agreement with experimental studies. However, the binding and the protein structure were significantly perturbed upon

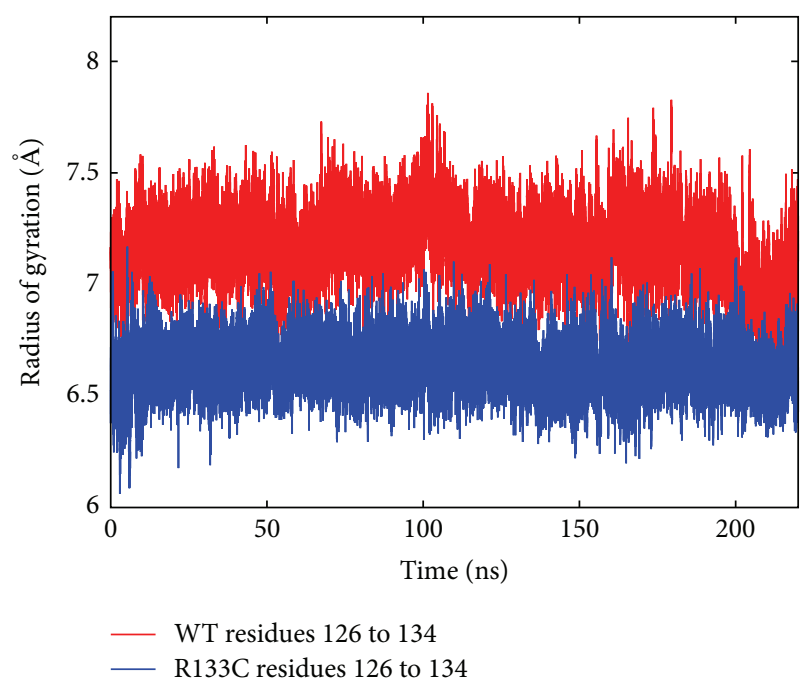

FIgURE 6: Time-dependent $R_{\text {gyr }}$ profile of residues 126 to 134 for WT (red) and R133C (blue).

TABLE 3: Average electrostatic and van der Waals interaction energies between protein and DNA.

\begin{tabular}{lcc}
\hline \multicolumn{3}{c}{ Average interaction energy between protein \& DNA $(\mathrm{kcal} / \mathrm{mol})$} \\
& $\mathrm{WT}$ & $\mathrm{R} 133 \mathrm{C}$ \\
\hline Electrostatic & $-840 \pm 7$ & $-711 \pm 6$ \\
van der Waals & $-56.4 \pm 0.5$ & $-59.2 \pm 0.6$ \\
\hline
\end{tabular}

mutation. Two hydrogen bonds that residue 133 makes with mDNA were lost upon the $\mathrm{R} 133 \mathrm{C}$ mutation, which may cause loss of specificity to mDNA in the mutant protein. Another important manifestation of the mutation is the reduced interaction energy between the mutant MBD and the DNA. Also, two salt bridges within the protein are lost upon the R133C mutation. The loss of salt bridges together with weakened interaction with DNA may cause a decrease in DNA-induced stability on MBD as the experiments suggest [15], which was found in our work to be manifested as the loss of helicity in the 141-142 region of mutant MBD. In addition, the protein hydration properties were significantly perturbed by R133C mutation. We speculate that the loss of helicity might have significant consequences in the interaction of $\mathrm{MeCP} 2$ with other protein partners subsequently adversely affecting its function, which may play a crucial role in RTT mechanism. Taken together, this suggests significant implications in understanding how the RTT-causing R133C mutation affects the protein structure at the molecular level. Further studies involving free energy calculations are underway to quantify the effects of this and other mutations on MBD of MeCP2-DNA interactions.

\section{Conflict of Interests}

The authors declare that there is no conflict of interests regarding the publication of this paper. 


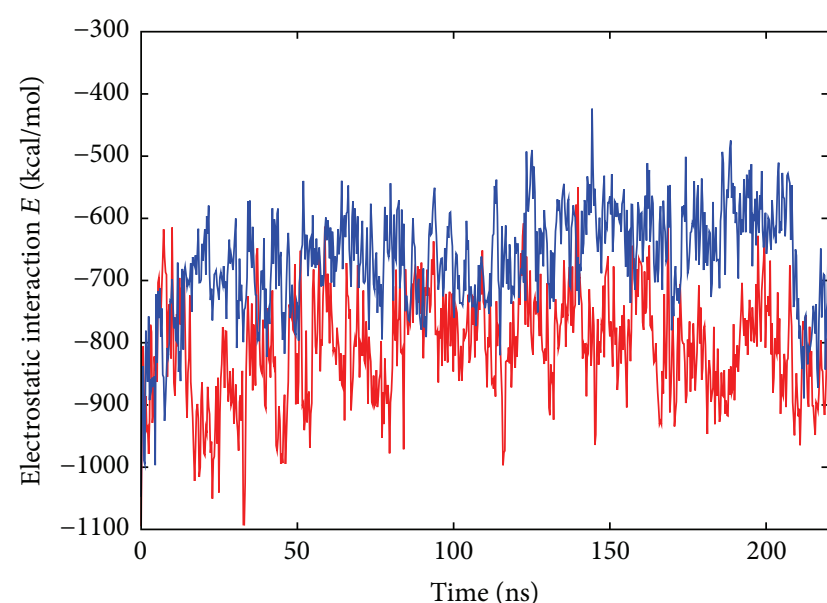

- WT

$-\mathrm{R} 133 \mathrm{C}$

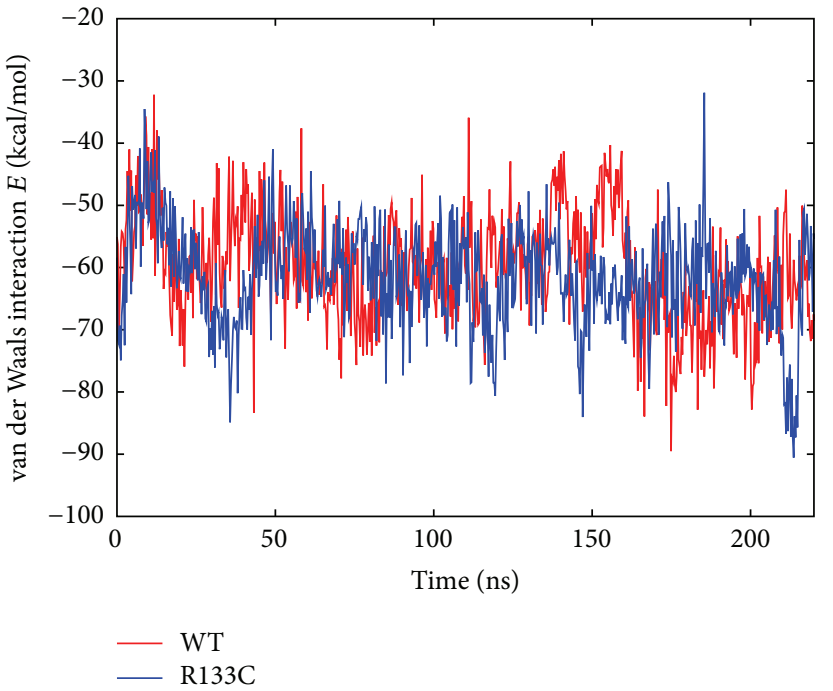

(b)

FIGURE 7: Electrostatic (a) and van der Waals (b) interaction energies between WT and DNA (red) and also between R133C and DNA (blue).

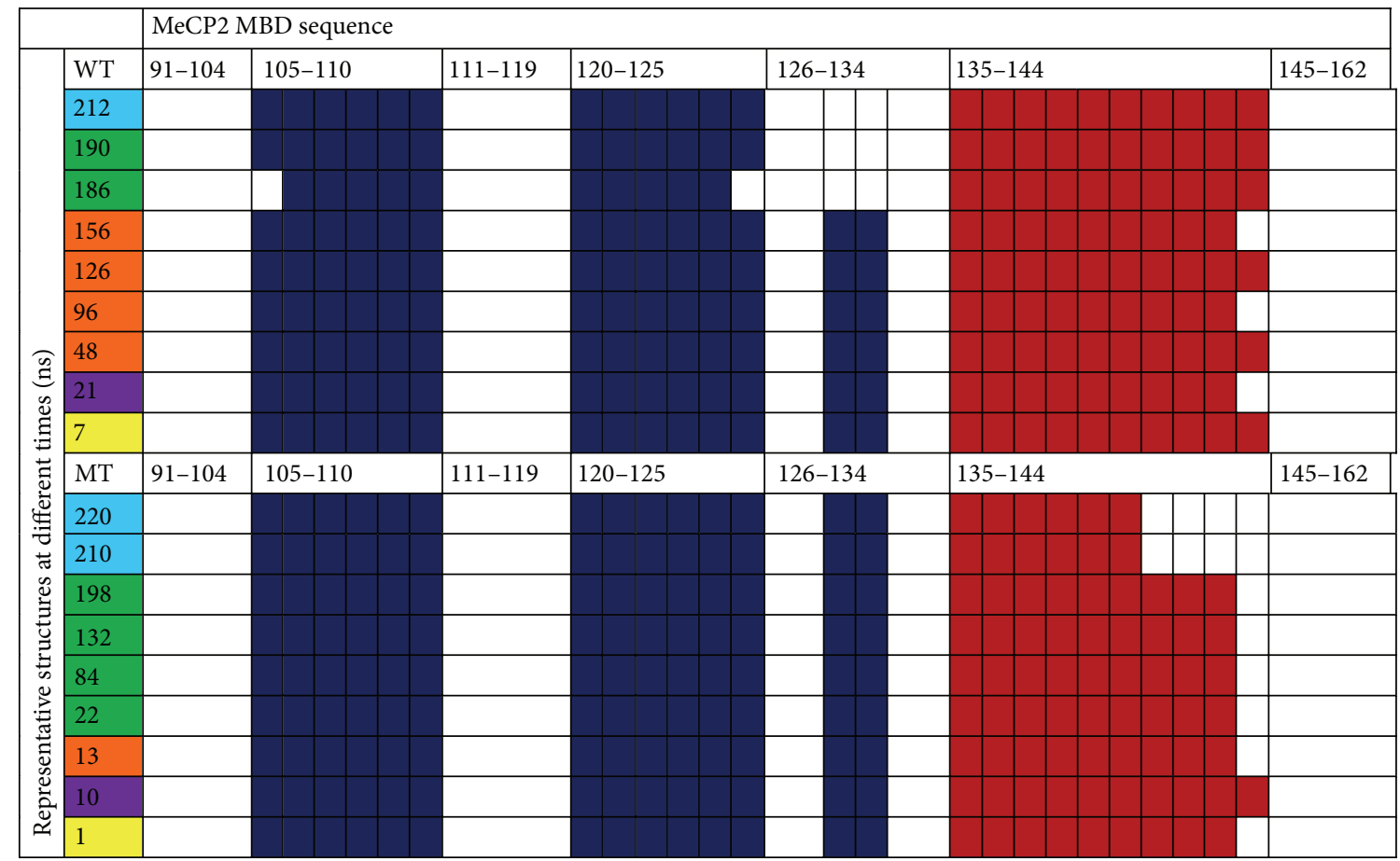

FIGURE 8: Secondary structure analysis for WT (top) and R133C (bottom). The left column shows representative structures at different stages (ns) of the simulation. The blue and red shaded areas represent strand and helix regions.

\section{References}

[1] D. D. Armstrong, "The neuropathology of Rett syndromeoverview 1994," Neuropediatrics, vol. 26, no. 2, pp. 100-104, 1995.

[2] B. Hagberg, "Clinical manifestations and stages of Rett syndrome," Mental Retardation and Developmental Disabilities Research Reviews, vol. 8, no. 2, pp. 61-65, 2002.

[3] S. B. Naidu, "Rett Syndrome," Indian Journal of Pediatrics, vol. 64, no. 5, pp. 651-659, 1997.
[4] B. A. Hagberg, "Rett syndrome: clinical peculiarities, diagnostic approach, and possible cause," Pediatric Neurology, vol. 5, no. 2, pp. $75-83,1989$.

[5] B. Hendrich and W. Bickmore, "Human diseases with underlying defects in chromatin structure and modification," Human Molecular Genetics, vol. 10, no. 20, pp. 2233-2242, 2001.

[6] H. G. Dunn and P. M. MacLeod, "Rett syndrome: review of biological abnormalities," Canadian Journal of Neurological Sciences, vol. 28, no. 1, pp. 16-29, 2001. 
[7] H. G. Dunn, "Importance of Rett syndrome in child neurology," Brain and Development, vol. 23, supplement 1, pp. S38-S43, 2001.

[8] R. E. Amir and H. Y. Zoghbi, "Rett syndrome: methyl-CpGbinding protein 2 mutations and phenotype-genotype correlations," The American Journal of Medical Genetics, vol. 97, no. 2, pp. 147-152, 2000.

[9] S. Akbarian, "The neurobiology of Rett syndrome," Neuroscientist, vol. 9, no. 1, pp. 57-63, 2003.

[10] D. D. Armstrong, "Review of Rett syndrome," Journal of Neuropathology and Experimental Neurology, vol. 56, no. 8, pp. 843-849, 1997.

[11] R. M. Zachariah and M. Rastegar, "Linking epigenetics to human disease and rett syndrome: the emerging novel and challenging concepts in MeCP2 research," Neural Plasticity, vol. 2012, Article ID 415825, 10 pages, 2012.

[12] J. L. Neul and H. Y. Zoghbi, "Rett syndrome: a prototypical neurodevelopmental disorder," Neuroscientist, vol. 10, no. 2, pp. 118-128, 2004.

[13] R. J. Klose, S. A. Sarraf, L. Schmiedeberg, S. M. McDermott, I. Stancheva, and A. P. Bird, "DNA binding selectivity of MeCP2 due to a requirement for $\mathrm{A} / \mathrm{T}$ sequences adjacent to methylCpG," Molecular Cell, vol. 19, no. 5, pp. 667-678, 2005.

[14] V. H. Adams, S. J. McBryant, P. A. Wade, C. L. Woodcock, and J. C. Hansen, "Intrinsic disorder and autonomous domain function in the multifunctional nuclear protein, MeCP2," The Journal of Biological Chemistry, vol. 282, no. 20, pp. 1505715064, 2007.

[15] R. P. Ghosh, R. A. Horowitz-Scherer, T. Nikitina, L. M. Gierasch, and C. L. Woodcock, "Rett syndrome-causing mutations in human MeCP2 result in diverse structural changes that impact folding and DNA interactions," The Journal of Biological Chemistry, vol. 283, no. 29, pp. 20523-20534, 2008.

[16] J. Christodoulou, A. Grimm, T. Maher, and B. Bennetts, "RettBASE: the IRSA MECP2 variation database-a new mutation database in evolution," Human mutation, vol. 21, no. 5, pp. 466472, 2003.

[17] N. C. Schanen, "Molecular approaches to the Rett syndrome gene," Journal of Child Neurology, vol. 14, no. 12, pp. 806-814, 1999.

[18] S. P. Chandler, D. Guschin, N. Landsberger, and A. P. Wolffe, "The methyl-CpG binding transcriptional repressor MeCP2 stably associates with nucleosomal DNA," Biochemistry, vol. 38, no. 22, pp. 7008-7018, 1999.

[19] K. L. Ho, I. W. McNae, L. Schmiedeberg, R. J. Klose, A. P. Bird, and M. D. Walkinshaw, "MeCP2 binding to DNA depends upon hydration at methyl-CpG," Molecular Cell, vol. 29, no. 4, pp. 525-531, 2008.

[20] W. L. Jorgensen, J. Chandrasekhar, J. D. Madura, R. W. Impey, and M. L. Klein, "Comparison of simple potential functions for simulating liquid water," The Journal of Chemical Physics, vol. 79, no. 2, pp. 926-935, 1983.

[21] W. Humphrey, A. Dalke, and K. Schulten, "VMD: visual molecular dynamics," Journal of Molecular Graphics, vol. 14, no. 1, pp. 33-38, 1996.

[22] A. D. MacKerell Jr., D. Bashford, M. Bellott et al., "All-atom empirical potential for molecular modeling and dynamics studies of proteins," Journal of Physical Chemistry B, vol. 102, no. 18, pp. 3586-3616, 1998.

[23] A. D. MacKerell Jr., M. Feig, and C. L. Brooks III, "Improved treatment of the protein backbone in empirical force fields,"
Journal of the American Chemical Society, vol. 126, no. 3, pp. 698-699, 2004.

[24] A. D. Mackerell Jr., M. Feig, and C. L. Brooks III, "Extending the treatment of backbone energetics in protein force fields: limitations of gas-phase quantum mechanics in reproducing protein conformational distributions in molecular dynamics simulation," Journal of Computational Chemistry, vol. 25, no. 11, pp. 1400-1415, 2004.

[25] N. Foloppe and A. D. MacKerell, "All-atom empirical force field for nucleic acids. I. Parameter optimization based on small molecule and condensed phase macromolecular target data," Journal of Computational Chemistry, vol. 21, no. 2, pp. 86-104, 2000.

[26] A. D. MacKerell Jr. and N. K. Banavali, "All-atom empirical force field for nucleic acids: II. Application to molecular dynamics simulations of DNA and RNA in solution," Journal of Computational Chemistry, vol. 21, no. 2, pp. 105-120, 2000.

[27] T. Darden, D. York, and L. Pedersen, "Particle mesh ewald: an $N \cdot \log (N)$ method for ewald sums in large systems," The Journal of Chemical Physics, vol. 98, no. 12, pp. 10089-10092, 1993.

[28] G. J. Martyna, D. J. Tobias, and M. L. Klein, "Constant pressure molecular dynamics algorithms," The Journal of Chemical Physics, vol. 101, no. 5, pp. 4177-4189, 1994.

[29] S. E. Feller, Y. Zhang, R. W. Pastor, and B. R. Brooks, "Constant pressure molecular dynamics simulation: the Langevin piston method," The Journal of Chemical Physics, vol. 103, no. 11, pp. 4613-4621, 1995.

[30] H. C. Andersen, "Rattle: a 'velocity' version of the shake algorithm for molecular dynamics calculations," Journal of Computational Physics, vol. 52, no. 1, pp. 24-34, 1983.

[31] J. C. Phillips, R. Braun, W. Wang et al., "Scalable molecular dynamics with NAMD," Journal of Computational Chemistry, vol. 26, no. 16, pp. 1781-1802, 2005.

[32] W. Kabsch and C. Sander, "Dictionary of protein secondary structure: pattern recognition of hydrogen-bonded and geometrical features," Biopolymers-Peptide Science Section, vol. 22, no. 12, pp. 2577-2637, 1983.

[33] G. Vriend, "WHAT IF: a molecular modeling and drug design program," Journal of Molecular Graphics, vol. 8, no. 1, pp. 52-56, 1990.

[34] T. M. Yusufzai and A. P. Wolffe, "Functional consequences of Rett syndrome mutations on human MeCP2," Nucleic Acids Research, vol. 28, no. 21, pp. 4172-4179, 2000. 


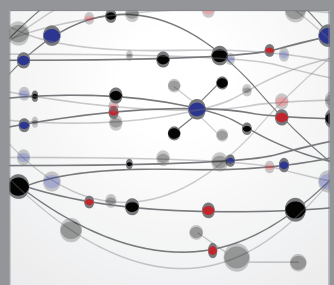

The Scientific World Journal
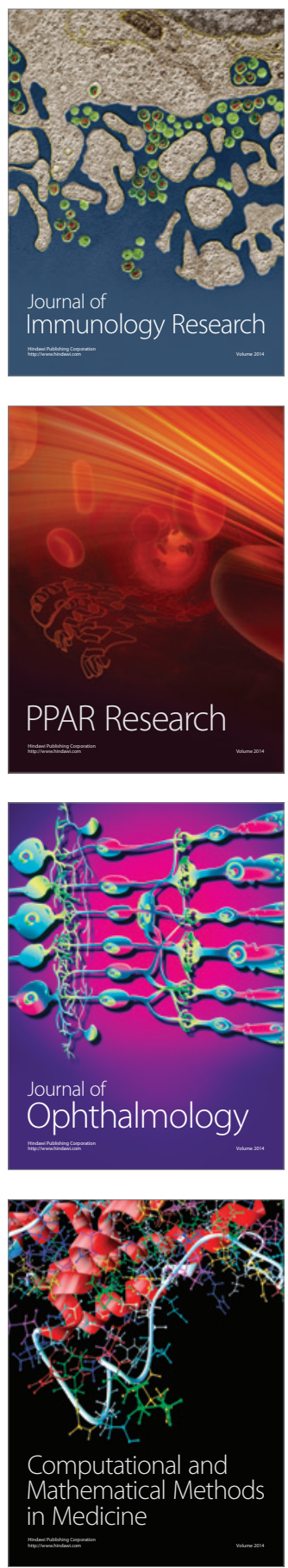

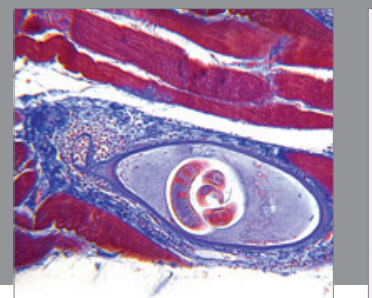

Gastroenterology

Research and Practice
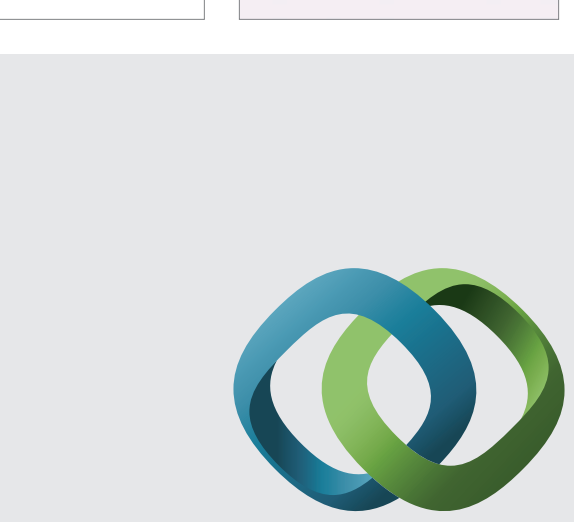

\section{Hindawi}

Submit your manuscripts at

http://www.hindawi.com
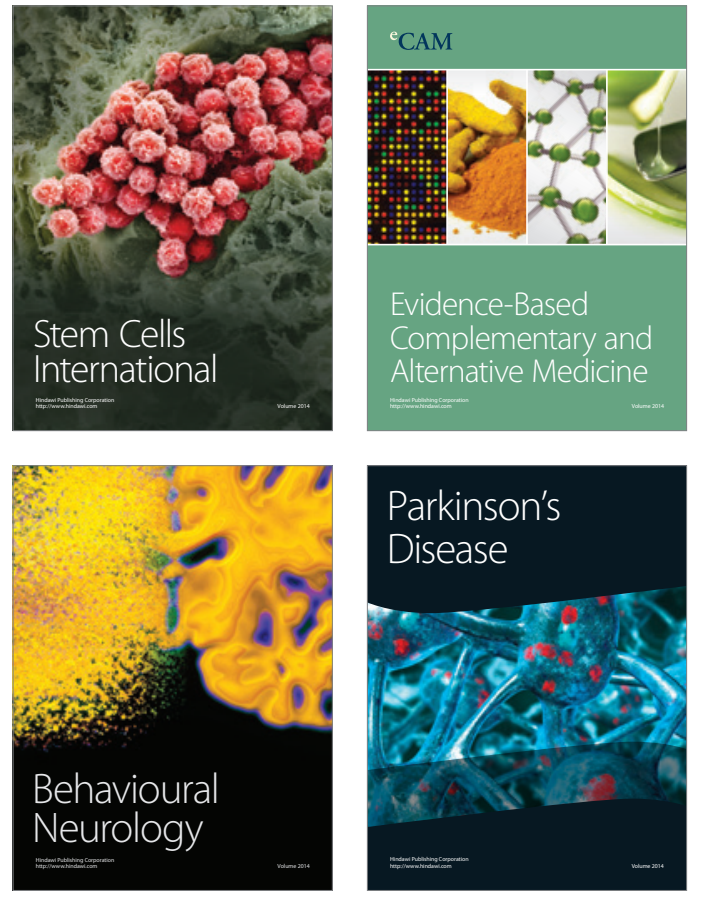
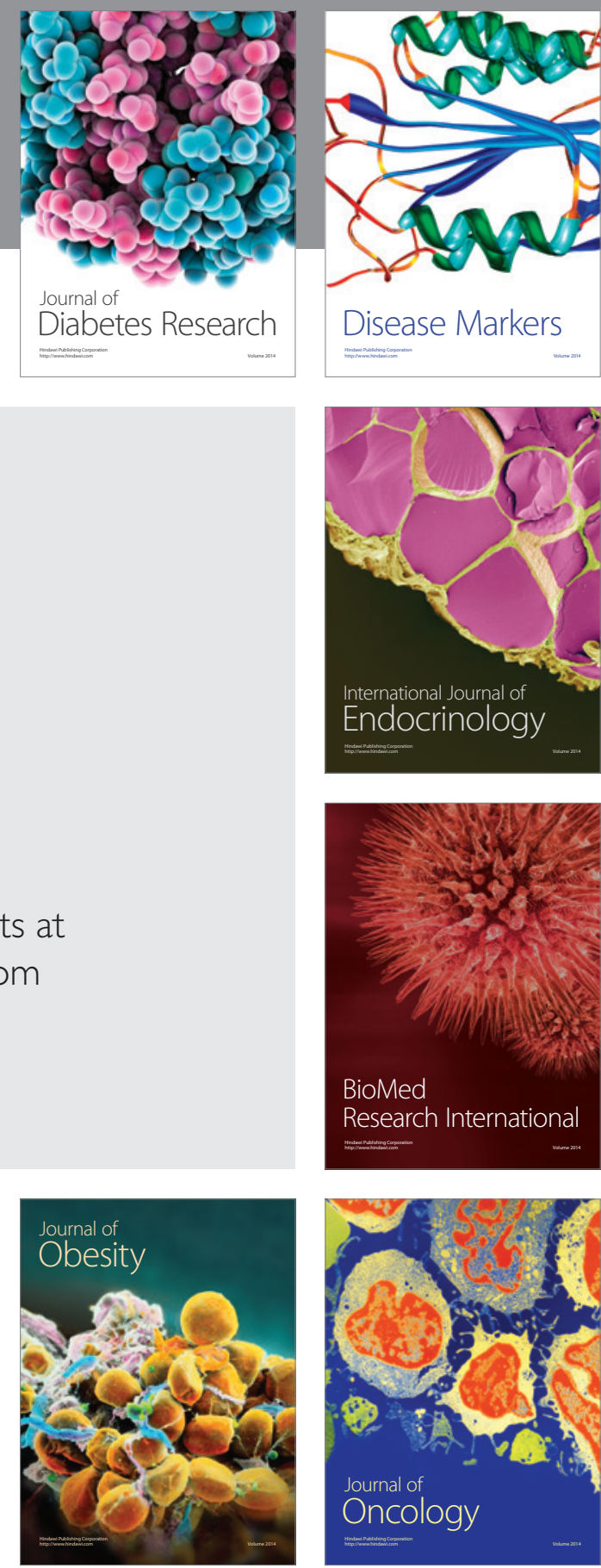

Disease Markers
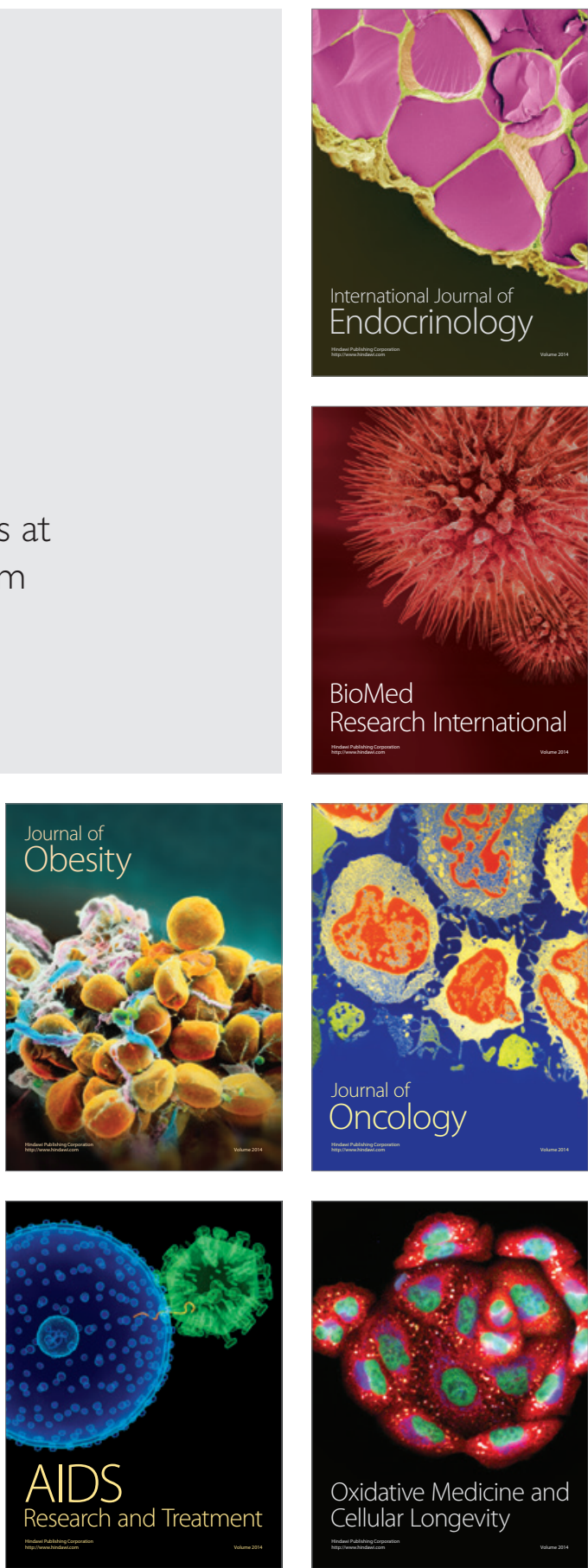